\title{
Escape of the Atmosphere of Rotating Planet
}

\author{
M.K.M. Ahmed and Z.M. Hayman*
}

Astronomy \& Meteorology Department, Faculty of Science, Cairo University, Giza, Egypt, 12613

\begin{abstract}
The problems of escape of a planetary atmosphere is reviewed. Formulae are derive for the rates of loss of mass and angular momentum from a unit area and from the whole planetary surface. The resulting formulae are applied to find the residence times of $\mathrm{H}, \mathrm{O}, \mathrm{N}_{2}, \mathrm{O}_{2}$ and $\mathrm{CO}_{2}$ in Martian atmosphere. All constituents are so stable while hydrogen is never stable and cannot be retained to my extent.
\end{abstract}

Keywords: Escape of a planetary atmosphere, loss of mass-residence times.

\section{INTRODUCTION}

The classical theory of escape has been developed by [1] who found that an atmosphere will be stable for astronomical periods (greater than $10^{9}$ years) if the root mean square velocity of the particle is less than the escape velocity $V_{e}$. Valuable discussions were given by [2-4]. [5] treated the problem taking into account the effect of orbital motion. The effect of rotation of the planet was introduced by [6] who derived formulae for the rates of loss of mass and angular momentum per unit area of the surface. In this paper formulae are derived to find expressions for the rates of loss of mass and angular momentum per unit area $f$ the surface (including higher order terms than obtained by Burke to applicable for higher rotational velocities), and for the total rates of loss of mass and angular momentum from the whole planetary surface.

The two necessary conditions for escape are:

1) the spatial velocity $V_{s}$ should be larger than the escape velocity $V_{e}$.

2) Negligible chance of undergoing collisions. This condition is satisfied at the critical level (base of the exosphere).

If $r_{\mathrm{o}}$ is the radius of the planet, the critical velocity required for the outgoing particles at a height $z$ above the planet's surface to escape is

$V_{e}=r_{o}\left(2 g_{o} / r\right)^{1 / 2}$

Where $g_{\mathrm{o}}$ is the gravity at the level $r_{\mathrm{o}}$ and $r=z+r_{\mathrm{o}}$

The rotation of the planet has two effects on the flux of the outgoing particles, (i) the reduction of gravity by the induced centrifugal force, resulting in an effective velocity of escape given by $V_{e}^{\prime 2}=V_{e}^{2}-V_{r}^{2}$; and (ii) particles in the same direction as the rotational motion will have more chance to escape than those in the opposite direction. Thus in addition to the most energetic particles those with the greatest forward angular momentum will have also increased

*Address for correspondence to this author at the Astronomy \& Meteorology Department, Faculty of Science, Cairo Universiy, Giza, Egypt; E-mail: zmhayman@gmail.com chance to attain escape speeds, resulting in an escaping flux primarily in the forward direction.

\section{Rates of Loss of Mass and Angular Momentum}

Consider a system of rectangular coordinates $\left(x_{1}, x_{2}, x_{3}\right)$. Let the plane $x_{1} x_{2}$ be tangent to the planetary surface with $x_{2}$ in the direction of the velocity of rotation $V_{r}$, and $x_{3}$ normal to the planetary surface. The rate of loss of mass per unit area will be

$$
\frac{d m}{d t}=\iiint_{\mu N}(V) V_{3} d V
$$

where $\mu$ is the mass per particle, $N(V) d V$ is the number of particles at the critical level $\left(r_{c}\right)$ with velocities between $V$ and $V+d V$.

The rate of loss of angular momentum is

$\frac{d l}{d t}=\iiint_{\mu N}(V)\left(V_{r}+V_{2}\right) R V_{3} d V$

$\mathrm{R}$ is the normal distance between the direction of $V_{2}$ and the axis of rotation (i.e. the radius of the parallel of latitude at the point over which escape is considered but measured to the vertical height $\left.z_{c}\right) . N(V) d V$ will be taken as maxwellian distribution function

$N(V) d V=\frac{n_{c}}{I I^{3 / 2} U^{3}} \exp \left(-\frac{V^{2}}{U^{2}}\right) d V_{1} d V_{2} d V_{3}$

$\mathrm{U}=\left(\frac{2 k T_{c}}{\mu}\right)^{\frac{1}{2}}=0.12895\left(\frac{T_{c}}{\mu^{\prime}}\right)^{\frac{1}{2}} \mathrm{~km} \cdot \mathrm{s}^{-1}$

where $n_{c}$ and $T_{c}$ are the total number of particles and temperature respectively at the critical level, and $\mu^{\prime}$ is molecular weight expressed in grams.

Transforming to a polar coordinate system at rest with respect to the center of the planet and with origin momentarily coincident with the former, we get.

$V_{1}=V_{s} \sin \theta \cos \varphi$,

$V_{2}=V_{s} \sin \theta \sin \varphi-V_{r}$, 
$V_{3}=V_{s} \cos \theta$.

and the distribution function becomes

$$
\begin{aligned}
N(V) d V= & \frac{n_{c}}{I I^{3 / 2} U^{3}} \exp \left[\left(-V_{s}^{2}+2 V_{r} V_{s} \sin \theta \sin \varphi-V_{r}^{2}\right) / U^{2}\right] \\
& . V_{s}^{2} \sin \theta d V_{s} d \theta d \varphi
\end{aligned}
$$

Substituting the distribution function given by eqn. (6) in eqns. (2) and (3) we get

$\frac{d m}{d t}=\frac{\mu n_{c}}{I I^{3 / 2}} U I_{m}$

and

$\frac{d l}{d t}=\frac{\mu n_{c}}{I I^{3 / 2}} R U I_{1}$

where

$I_{m}=\exp \left(-u_{r}^{2}\right) \exp \left(-u_{s}^{2}+2 u_{r} u_{s} \sin \theta \sin \varphi\right)$

$u_{s}^{3} \cos \theta \sin \theta \int_{u_{e}}^{\infty} d u_{s} \int_{0}^{2 \pi} d \varphi \int_{0}^{2 \pi} d \theta$

$I_{1}=\exp \left(-u_{r}^{2}\right) \exp \left(-u_{s}^{2}+2 u_{r} u_{s} \sin \theta \sin \varphi\right)$

$u_{s}^{4} \cos \theta \sin \theta \int_{u_{e}}^{\infty} d u_{s} \int_{0}^{2 \pi} d \varphi \int_{0}^{2 \pi} d \theta$

and

$u_{s}=V_{s} / U, u_{e}=V_{e} / U, u_{r}=V_{r} / U$

It is clear that the rotational velocity is contained only in $u_{r}$. Considering $u_{r}$ as a measure of the effect of rotaion on the escape process, we can divide the planets into three groups:

Planets for which rotation can be neglected, these are Mercury and Venus

(ii) Planets for which $u_{r}<<1$ and hence higher powers of $u_{r}$ can be neglected these are the Earth and Mars.

(iii) Planets with $u_{r}>>1$, these are the major planets.

Considering the case when $u_{r} \rightarrow 0$, eqns. (9) and (10) can be integrated easily to yield

$\operatorname{Im}=\frac{\pi}{2} \exp \left(-u_{e}^{2}\right)\left(1+u_{e}^{2}\right)$

and

$I_{1}=0$

The Value of $I_{1}=0$ is clear since we have assumed in advance that the planet does not rotate. The first of eqns. (12), combined with eqn. (7) give the rate of loss of mass per unit area when the planetary rotation is negligible.

Considering the second case, i.e. $u_{r}<<1$. Set $\mathrm{y}=\sin \varphi$, and $\mathrm{b}=2 u_{r} u_{s}$. Since $u_{s} \approx 1$, then $b<1$. Carrying out the $\theta$ interaction, eqns (9) and (10) yield

$I_{m}=\exp \left(-u_{r}^{2}\right) \int_{u_{e}}^{\infty} d u_{s} \int_{0}^{2 \pi} d \varphi \exp \left(-u_{s}^{2}\right) u_{s}^{3} \mathrm{f}_{m}$

and
$I_{1}=\exp \left(-u_{r}^{2}\right) \int_{u_{e}}^{\infty} d u_{s} \int_{0}^{2 \pi} d \varphi u_{s}^{2} \exp \left(-u_{s}^{2}\right) u_{s}^{3} \mathrm{f}_{1}$

where

$\mathrm{f}_{m}=\sum_{n=0} \frac{1}{(n+2) n !} b^{n} y^{n}$

$\mathrm{f}_{1}=\sum_{n=0} \frac{1}{(n+3) n !} b^{n} y^{n+1}$

Substituting $f_{m}$ and $f_{1}$ in (13) and (14) respectively, retaining only terms up to the fifth power in $\mathrm{b}$ and $u_{r}$, writing $\varphi$ sinfor $y$ and performing the $\varphi$ and $u_{s}$ integration result in

$I_{m}=\frac{I I}{2} \exp \left(-u_{e}^{2}\right)\left\{1+u_{e}^{2}+\frac{1}{2} u_{r}^{2} u_{e}^{4}-\frac{1}{4} u_{r}^{4} u_{e}^{4}\left(1-\frac{1}{3} u_{e}^{2}\right)\right\}$

and

$I_{1}=\frac{I I}{2} \exp \left(-u_{e}^{2}\right) u_{r}\left\{1+u_{e}^{2}+\frac{1}{2} u_{e}^{4}+\frac{1}{6} u_{r}^{2} u_{e}^{6}-\frac{1}{2} u_{r}^{4} u_{e}^{6}\left(1-\frac{1}{4} u_{e}^{2}\right)\right\}$

It is clear that in the limit as $u_{r} \rightarrow 0$, eqns. (17) and (18) reduce to eqns. (12). Neglecting terms of order higher than the second in $u r$ we obtain those of [6].

In the third case $u_{r}>>1$ we can not expand $\mathrm{f}_{m}$ and $\mathrm{f}_{1}$ in powers of $u_{r}$ and hence eqns. (13) and (14) have to be integrated numerically.

\section{Total Rates of Loss from the Whole Surface}

Assuming that $u_{r}$ is computed at the equator $r=r_{o}$, then at any other latitude $\mathrm{D}$ its value will be $u_{r} \cos D$. The rates of loss from circular element of the surface parallel to the equator and of width $r_{o} d D$ will be given by f.cos $2 I I r_{o}^{2} D$ $d D$. f, where f represents $d m / d t$ or $d l / d t$. Further

$R=\left(r_{o}+z\right) \cos D$

where $z$ is the height of the following particle. It is to be noted that for a particle of height $z, u_{r}$ may be larger than that of the same thermal velocity. But since the particle may not be strictly constrained to rotate with the same angular velocity as the planet, it seems sufficient to consider the value of $u_{r}$ at the surface.

The total rates of mass and angular momentum from the whole planet will be

$\frac{d M}{d t}=\frac{4 n}{I I^{1 / 2}} u r_{o}^{2} I_{M}$
$\frac{d L}{d t}=\frac{4 n}{I I^{3 / 2}} u^{2} r_{o}^{2}\left(r_{0}+z\right) I_{L}$

where

$$
\begin{aligned}
& I_{M} \int_{0}^{\pi / 2} I_{m}\left(u_{r} \quad \cos D\right) \cos D d D \\
& I_{L} \int_{0}^{\pi / 2} I_{l}\left(u_{r} \quad \cos D\right) \cos ^{2} D d D
\end{aligned}
$$

In case of large rotational velocities $u_{r}>>1,\left(u_{r} \cos D\right)$ and $I_{l}\left(u_{r}, \cos D\right)$ are first obtained by numerical integration of formulae (13) and (14) for different values of $D$ (i.e. for 
values of $u_{r} \cos D$ from 0 , at the pole, up to $u_{r}$, at the equator) and then performing numerically the integrations in equations (21).

When $u_{r}<1$, substituting from eqns. (17) and (18) in eqns. (21) and integrating we get

$I_{M}=\frac{I I}{2} \exp \left(-u_{e}^{2}\right)\left\{1+u_{e}^{2}+\frac{1}{3} u_{r}^{2} u_{e}^{4}-\frac{2}{15} u_{r}^{4} u_{e}^{4}\left(1-\frac{1}{3} u_{e}^{2}\right)\right\}$

$I_{L}=\frac{I I}{3} \exp \left(-u_{e}^{2}\right) u_{r}\left\{1+u_{e}^{2}+\frac{1}{2} u_{e}^{4}+\frac{2}{15} u_{r}^{2} u_{e}^{6}-\frac{2}{35} u_{r}^{4} u_{e}^{6}\left(1-\frac{1}{4} u_{e}^{2}\right)\right\}$

Again the classical formulae is obtained by neglecting terms including $u_{r}$ :

$I_{M}=\frac{I I}{2} \exp \left(-u_{e}^{2}\right)\left(1+u_{e}^{2}\right)$

Eqns. (20), (22), and (23) give the total rates of loss of mass and angular momentum from the whole planet assuming that colisions beyond the critical level, at $r_{c}$, are negligible, otherwise they will merely represent the flux of outgoing particles. The escaping particles are provided by diffusion from below, if the rate of escape is much higher than that of diffusion, then diffusion will control the rate of escape. Further, the net rate of loss will be smaller than those given by the above eqns. due to the input of particles from the interplanetary medium in addition to the existence of some collisions above the critical level which cause some particles to return back below critical level. Also near the critical level collisions become so rare that Maxwellian distribution will hold less exact.

\section{HISTORY OF MARS ATMOSPHERE}

It is widely belived that the climate on Mars was much different than the present one $[7,8]$. The criteria led to this belief :

(1) Valley networks suggest that liquid water was once existant $[9,10]$,

There are indications that erosion rates 3.5-4 Gyrs. ago was much higher than it is now.

For the erosion to be effective, liquid water must exist on surafce, while the present mean equatorial temperature (about $250^{\circ} \mathrm{K}$ ) and the mean surface pressure $(6-7 \mathrm{mb}$ ) cause ice to sublime without passing with the liquid state. Hence the surface pressure at some early stage must have been above $30 \mathrm{mb}$ [11]. The importance of this point increases by the presence of evidences from the degradation of impact crateres on the oldest surfaces which suggests that erosion processes were more efficient up untill 3.5 by age [12].

Some authers go to saying that the Mars early climate even had some similarity to the earth's climate at the epoch when life was first forming, and as the solar output was their loss by $30 \%$ than at present [13] this opened the posibility that life may have formed on early Mars [14, 15].

Conducted a 3-dimensional simulation of HDO in the Mars atmosphere which has important clues to understanding climate evolution [16]. The study revealed that the Mars atmosphere is, at present enriched in denterium concentration providing an indirect evidence of a metter climate in the past.

If the above were true, that is the early Mars climate was substantially different from the presently existing one then this would be a large evidence that the atmospheric constituents undergo much time variations and points to the importance of analyzing the important loss and regeneration processes. These can be outlimed as: Impact erosion [17], Sputtering by Solar wind [18, 19], Weathering [11], incorporation in the polar caps and like formations [20], and photochemical processes. Clearly if we stress only in the loss processes, these means become minor component to the escape first outgoing particles, which is object of present work.

\section{THE RESIDENCE TIME}

The residence time $t$ may be defined as the time interval during which a certain constituents decreases by factor $\mathrm{e}^{-1}$, where $\mathrm{e}$ is the base of Napierian logrithms.

If $\mathrm{N}_{\mathrm{o}}$ is the total number of particles at time $\mathrm{t}_{0}$, and $\mathrm{F}$ the escaping flux then

$t=\frac{N_{o}}{F}$

\section{THE CRITICAL LEVEL}

The height $z_{c}$ of the critical level can be determined from the condition that a fraction $\mathrm{e}^{-1}$ of the fast upward moving particles at $\mathrm{z}_{\mathrm{c}}$ will experince no collisions during their ascent, that is, the probability of collisions equals e- 1 .

With this definition, the height of the critical level is that at which

$n_{c}=\left(4 I I^{a} 2 H_{c}\right)^{-1}$

where a is the radius of a representative particle level is that at which

$H_{c}=R T / \mu g$

Since the mean free path is proportional to $\left(4 I I^{a} 2 H_{c}\right)^{-1}$, we see that at the critical level the mean free path for the particle moving fast in a horizontal direction is equal to the scale height.

For Mars, a, can be taken as that of a molecule of $\mathrm{CO}_{2}$, or about $1.6 \times 10^{-8} \mathrm{~cm}$. Using data reported by [21], and computing the right and left hand sides of eqn. (25) for heights up to $260 \mathrm{kms}$. the critical level is estimated to occur at about $208 \mathrm{kms}$. where the temperature is about $400^{\circ} \mathrm{K}$ and the number density is $1.5 \times 10^{8} \mathrm{~cm}^{-3}$.

\section{ESCAPE OF MARS ATMOSPHERIC CONSTITUENTS}

In this section the escape of $\mathrm{H}, \mathrm{O}, \mathrm{N}_{2}, \mathrm{O}_{2}$, and $\mathrm{CO}_{2}$ will be considered. The loss of mass and angular momentum and escape flux of particles per unit areas, and from the whole surface of the planet will be computed using formulae (7), (8), (17), (18), (20), and (22), and then the residence times of these constituents are computed using eqn. (24) and assuming, for simplicity, that the total number of particles is given by 
Table 1. The Results (The Residence Time)

\begin{tabular}{|c|c|c|c|c|c|}
\hline Gas & $\mathbf{H}$ & $\mathbf{O}$ & $\mathbf{N}_{2}$ & $\mathbf{O}_{2}$ & $\mathrm{CO}_{2}$ \\
\hline $\mathrm{n}_{\mathrm{c}}$ & $10^{7}$ & $10^{7}$ & $10^{7}$ & $10^{6}$ & $10^{8}$ \\
\hline $\mathrm{N}_{\mathrm{o}}$ & $1.4 \times 10^{34}$ & -- & $2.4 \times 10^{40}$ & $4.2 \times 10^{38}$ & $3 \times 10^{41}$ \\
\hline $\mathrm{dm} \backslash \mathrm{dt}$ & $3 \times 10^{3}$ & $16 \times 10^{-17}$ & $6.2 \times 10^{-35}$ & $8 \times 10^{-42}$ & $1.6 \times 10^{-56}$ \\
\hline $\mathrm{dM} \backslash \mathrm{dt}$ & $3 \times 10^{22}$ & 58 & $2 \times 10^{-16}$ & $2.6 \times 10^{-23}$ & $3.8 \times 10^{-39}$ \\
\hline $\mathrm{dL} \backslash \mathrm{dt}$ & $4 \times 10^{35}$ & $7.3 \times 10^{15}$ & $4 \times 10^{-2}$ & $5.8 \times 10^{-9}$ & $1.1 \times 10^{-24}$ \\
\hline $\mathrm{dn} \backslash \mathrm{dt}$ & $5 \times 10^{26}$ & $9.8 \times 10^{4}$ & $2.2 \times 10^{-13}$ & $2.5 \times 10^{-20}$ & $3.7 \times 10^{-35}$ \\
\hline $\mathrm{dN} \backslash \mathrm{dt}$ & $3 \times 10^{45}$ & $3.6 \times 10^{23}$ & $7.3 \times 10^{5}$ & $8.1 \times 10^{-2}$ & $8.5 \times 10^{-18}$ \\
\hline
\end{tabular}

$N_{o}=4 I I r^{2} n_{o} H_{o}$

where $n_{\mathrm{o}}$, and $H_{\mathrm{o}}$ are, respectively, the number density and scale height of each constituent near the surface.

The value of $n_{0}$, and the number densities of $\mathrm{O}, \mathrm{N}_{2}, \mathrm{O}_{2}$, and $\mathrm{CO}_{2}$ at the critical level are given by [21]; Tois taken equal $225^{\circ} \mathrm{K}$. The number density of Hydrogen molecules near the surface is assumed equal to the number of Oxygen atoms (about $10^{7} \mathrm{~cm}^{-3}$ ). The results are given in Table $\mathbf{1}$. Small letters are used to indicate loss from unit area (at the equator) and capital letters to indicate loss from the whole planetary surface. The residence time is in units of $10^{9} \mathrm{yrs}$. The table reveals while hydrogen is never stable and could not be retained to any extent.

Finally setting the effect of orbital motion to zero in [5] the present formulas result, indicating the validity of both.

\section{REFERENCES}

[1] Jeans J. Dynamical theory of gases. Cambridge University Press 1916.

[2] Spitzer L. The atmosphere of the Earth and planets. Kuiper ed. Chicago University Press 1952.

[3] MacDonald GJF. The origin and evaluation of atmospheres and oceans. Branz Cam Ed. Wiley Press 1964.

[4] Brandt JC. Hodge PW. Solar system astrophysics. McGraw Hill 1964.

[5] Ahmed MKM, Rassem M, Ismail MN. Escape of planetary atmospheres including the effects of rotation and orbital motion of the planet. Earth Moon Planets 1999; 84: 95-108.

[6] Burke TA. Evaporation from rotating planets. Mont Scat Rev astrn soc 1969; 145: 487.
[7] Squyres SW. Kasting JF. Early Mars: How warm and how wet? Science 1994; 265: 744.

[8] Jakosky B, Jones M. The history martian volatiles. Revs Geophys 1997; 35: 1 .

[9] Carr MH. Mars: A water-rich planet? ICARUS 1986; 68: 187.

[10] Carr MH. The Martian drainage system and the origin of valley networks and fretted channels. J Geophys Res 1995; 100: 4779.

[11] Haberle RM. Mars's volatile and climate history. J Geophys Res 1998; $103: 28467$.

[12] Craddock RA, Maxwell A. Geomorphic evolution of the Martian highlands through ancient fluvial processes. J Geophys Res 1993; 89: 3453 .

[13] Kasting JF. $\mathrm{CO}_{2}$ condensation and the climate of early Mars. ICARUS 1991; $94: 1$

[14] Mckay CP, Stoker CR. The early environment and its. evolution on Mars: Implications for life. Rev Geophys 1989; 27: 189

[15] Mckay CP, Gibson Jr, Thomas-Keprta KL, et al. Search for past life on Mars: Possible relic biogenic activity in martian meteorite ALH84001. Science 1996; 273: 924.

[16] Montmessin F, Fouchet T, Forget F. Modelling the annual cycle of HDO in the martian atmosphere. J Geophys Res 2005; 110: E03006.

[17] Hunten DM. Atmosphere evolution of the terrestrial planets. Science 1993; 259: 915.

[18] Jakosky BM, Pepln RO, Fox JL. Mars atmospheric loss and isotopic fractionation by solar-wind-induced sputtering and photochemical escape. ICARUS 1994; 315: 559.

[19] Samuel E. Planets like Earth are probably common place in the Universe, reveals a stellar analysis. New Sci 2001; 169: 2227.

[20] Jakosky BM, Carr MH. Possible precipitation of ice at low latitudes of Mars during periods of high obliquity. Nature 1985; 315: 559 .

[21] Ahmed MKM. Planetary atmosphere with special reference to Mars. M.Sc.Thesis Cairo University; 1973.

(C) Ahmed and Hayman; Licensee Bentham Open.

This is an open access article licensed under the terms of the Creative Commons Attribution Non-Commercial License (http://creativecommons.org/licenses/bync/3.0/), which permits unrestricted, non-commercial use, distribution and reproduction in any medium, provided the work is properly cited. 